ELECTRONIC RESEARCH ANNOUNCEMENTS OF THE AMERICAN MATHEMATICAL SOCIETY

Volume 12, Pages 37-43 (March 31, 2006)

S $1079-6762(06) 00158-2$

\title{
PICARD-HAYMAN BEHAVIOR OF DERIVATIVES OF MEROMORPHIC FUNCTIONS WITH MULTIPLE ZEROS
}

\author{
SHAHAR NEVO, XUECHENG PANG, AND LAWRENCE ZALCMAN \\ (Communicated by Svetlana Katok)
}

\begin{abstract}
The derivative of a transcendental meromorphic function all of whose zeros are multiple assumes every nonzero complex value infinitely often.
\end{abstract}

In 1959, Hayman [3] proved the following seminal result, which has come to be known as Hayman's Alternative.

Theorem A. Let $f$ be a transcendental meromorphic function on the complex plane C. Then either

(i) $f$ assumes each value $a \in \mathbb{C}$ infinitely often, or

(ii) $f^{(k)}$ assumes each value $b \in \mathbb{C} \backslash\{0\}$ infinitely often for $k=1,2, \ldots$.

Considering the function $g(z)=[f(z)-a] / b$ shows that it suffices to take $a=0$ and $b=1$ in Theorem A.

Associated with Theorem A are the following companion results.

Theorem B. Let $f$ be a meromorphic function on $\mathbb{C}$. If $f(z) \neq 0$ and $f^{(k)}(z) \neq 1$ for some fixed positive integer $k$ and all $z \in \mathbb{C}$, then $f$ is constant.

Theorem C. Let $\mathcal{F}$ be a family of meromorphic functions on a plane domain $D$. Suppose that for each $f \in \mathcal{F}, \quad f(z) \neq 0$ and $f^{(k)}(z) \neq 1$ for some fixed positive integer $k$ and all $z \in D$. Then $\mathcal{F}$ is a normal family on $D$.

Theorem B is an immediate consequence of Theorem A, which shows that no transcendental meromorphic function can satisfy $f(z) \neq 0, f^{(k)}(z) \neq 1$ for all $z \in \mathbb{C}$. On the other hand, if $f$ is a nonconstant rational function such that $f(z) \neq 0$ for $z \in \mathbb{C}$, then $f(\infty)=0$ for each $k \geq 1$, so $f^{(k)}$ assumes every value with the possible exception of 0 in the finite plane. Theorem $\mathrm{C}$, a celebrated result of $\mathrm{Gu}$ [4], is related to Theorem B via Bloch's Principle [8, p. 222]; for a very simple proof along these lines, see [8, p. 225].

In recent years, it has become clear that, in many instances, the condition $f \neq 0$ can be replaced by the assumption that all zeros of $f$ have sufficiently high multiplicity. This announcement concerns such an extension of Theorem A. We restrict our attention to the case $k=1$.

Received by the editors November 23, 2005.

2000 Mathematics Subject Classification. Primary 30D35, 30D45.

Key words and phrases. Meromorphic functions, quasinormal families.

This work was supported by the German-Israel Foundation for Scientific Research and Development G.I.F. Grant No. I-809-234-6/2003.

(C)2006 American Mathematical Society Reverts to public domain 28 years from publication 


\section{WHAT WAS KNOWN BEFORE}

Before stating our result, let us indicate what has already been stated or proved. We have the following analogues of the results stated above.

Theorem $\mathbf{A}^{\prime}$. Let $f$ be a transcendental meromorphic function on $\mathbb{C}$, all of whose zeros have multiplicity at least 3 . Then $f^{\prime}$ assumes each nonzero complex value infinitely often.

Theorem $\mathbf{B}^{\prime}$. Let $f$ be a meromorphic function on $\mathbb{C}$, all of whose zeros have multiplicity at least 3 . If $f^{\prime}(z) \neq 1$ for all $z \in \mathbb{C}$, then $f$ is constant.

Theorem $\mathbf{C}^{\prime}$ (Wang and Fang [6]). Let $\mathcal{F}$ be a family of meromorphic functions on a plane domain D, all of whose zeros have multiplicity at least 3. If, for each $f \in \mathcal{F}, f^{\prime}(z) \neq 1$ for all $z \in D$, then $\mathcal{F}$ is normal on $D$.

Theorem $\mathrm{A}^{\prime}$ is stated as Theorem 3 (with $k=1$ ) in [6]. Although the proof indicated there is inadequate, it is not difficult to base a proof on other results stated and proved in that paper. It follows from Theorem $\mathrm{A}^{\prime}$ that a function satisfying the hypotheses of Theorem $\mathrm{B}^{\prime}$ must be rational, and then Lemma 10 of [6] shows that it must be constant. Theorem $\mathrm{C}^{\prime}$ is Theorem 7 of [6] (with $n=3$ and $k=1$ ).

Theorems $\mathrm{B}^{\prime}$ and $\mathrm{C}^{\prime}$ are best possible in the sense that neither is true if 3 is replaced by 2 . Indeed, we have the following examples.

Example 1. Fix $a, b \in \mathbb{C}, a \neq b$. The function

$$
f(z)=\frac{(z-a)^{2}}{z-b}=z+(b-2 a)+\frac{(a-b)^{2}}{z-b}
$$

vanishes only at $z=a$, where it has a double zero. Clearly, $f^{\prime}(z) \neq 1$ for all $z \in \mathbb{C}$. Thus Theorem $\mathrm{B}^{\prime}$ fails when 3 is replaced by 2 .

Example 2. Let $\Delta=\{z:|z|<1\}$ and $\mathcal{F}=\left\{f_{\alpha}\right\}$, where

$$
f_{\alpha}(z)=\frac{(z-\alpha)^{2}}{z-2 \alpha}=z+\frac{\alpha^{2}}{z-2 \alpha}, \quad \alpha \in \mathbb{C} \backslash\{0\} .
$$

Then all zeros of $f_{\alpha}$ are multiple and $f_{\alpha}^{\prime}(z) \neq 1$ for $z \in \Delta$. However, $f_{\alpha}$ takes on the values 0 and $\infty$ in any fixed neighborhood of 0 if $\alpha$ is sufficiently small, so $\mathcal{F}$ fails to be normal at 0 . Thus Theorem $C^{\prime}$ does not hold with 3 replaced by 2 .

On the other hand, the analogue of Theorem $\mathrm{A}^{\prime}$ with 3 replaced by 2 does hold for functions of finite order (cf. Lemma 6 of [6]).

Theorem $\mathbf{A}^{\prime \prime}$. Let $f$ be a transcendental meromorphic function of finite order on $\mathbb{C}$, all of whose zeros are multiple. Then $f^{\prime}$ assumes each nonzero complex value infinitely often.

This is an instant corollary of Theorem A and the following important result.

Theorem D (Bergweiler and Eremenko [1]). Let $f$ be a transcendental meromorphic function of finite order on $\mathbb{C}$ with an infinite number of multiple zeros. Then $f^{\prime}$ assumes each nonzero complex value infinitely often.

Indeed, if in Theorem $\mathrm{A}^{\prime \prime}, f$ vanishes only finitely often, then $f^{\prime}$ must take on every nonzero value infinitely often by Theorem A; otherwise, Theorem D implies the same conclusion.

Theorem D is not true in general for functions of infinite order. 
Example 3 (Bergweiler and Eremenko [1]). Let

$$
f(z)=z+a \int_{0}^{z} \exp \left(b e^{\zeta}-\zeta\right) d \zeta
$$

where $1+a b=0$ and $1+a e^{b}=0$. (To find such $a$ and $b$, pick $b$ so that $e^{b}=b$ and set $a=-1 / b$.) Clearly, $f(0)=0$. Moreover, substituting $w=e^{\zeta}$ and using residues to evaluate the integral shows that $f(z+2 \pi i)-f(z)=2 \pi i(1+a b)=0$, so that $f$ has period $2 \pi i$. Since $f^{\prime}(z)=1+a \exp \left(b e^{z}-z\right), f^{\prime}(0)=1+a e^{b}=0$; hence, by periodicity, $f$ has multiple zeros at the points $2 \pi i k, k \in \mathbb{Z}$. But clearly, $f^{\prime}(z) \neq 1$.

\section{THE MAIN RESUlT}

Although Theorem D fails for functions of arbitrary (i.e., infinite) order, we do have the following extension of Theorems $\mathrm{A}^{\prime}$ and $\mathrm{A}^{\prime \prime}$.

Theorem 1. The derivative of a transcendental meromorphic function on $\mathbb{C}$ all of whose zeros are multiple assumes every nonzero complex value infinitely often.

As a simple consequence, we obtain the following result, first proved in [1], 2], and [7]; cf. [8, p. 226].

Theorem E. If $f$ is a transcendental meromorphic function on $\mathbb{C}$, then $f^{\prime} f^{n}$ takes on every nonzero complex value infinitely often for each $n \geq 1$.

Indeed, $f^{n+1}$ has only multiple zeros and $\left(f^{n+1}\right)^{\prime}=(n+1) f^{\prime} f^{n}$.

\section{How not to PRove Theorem 1}

An outline of the proof of Theorem A' (assuming other results stated above) will illustrate the difficulty involved in proving Theorem 1. Suppose then, that E $f$ is a transcendental meromorphic function on $\mathbb{C}$ all of whose zeros have multiplicity at least 3, and that $f^{\prime}$ assumes some nonzero value, say 1 , at most finitely often. Then, by Theorem $\mathrm{A}^{\prime \prime}, f$ has infinite order. It follows (cf. [8, p. 217]) that the spherical derivative

$$
f^{\#}(z)=\frac{\left|f^{\prime}(z)\right|}{1+|f(z)|^{2}}
$$

is unbounded. Choose $z_{n}$ such that $f^{\#}\left(z_{n}\right) \rightarrow \infty$; then $z_{n} \rightarrow \infty$. Set $f_{n}(z)=$ $f\left(z+z_{n}\right)$. Clearly, all zeros of these functions on the unit disc $\Delta$ have multiplicity at least 3. Moreover, since $f^{\prime}$ assumes the value 1 only finitely often on $\mathbb{C}$ and $z_{n} \rightarrow \infty$, renumbering if necessary, we may assume that $f_{n}^{\prime}(z) \neq 1$ for all $n$ and all $z \in \Delta$. Then, by Theorem $\mathrm{C}^{\prime}$, the family $\mathcal{F}=\left\{f_{n}\right\}$ is normal on $\Delta$. On the other hand, $f_{n}^{\#}(0)=f^{\#}\left(z_{n}\right) \rightarrow \infty$, so by Marty's theorem (cf. [8, p. 216]), $\mathcal{F}$ is not normal on $\Delta$. This contradiction proves Theorem $\mathrm{A}^{\prime}$. (A more complicated argument can be used to derive a contradiction from Theorem $\mathrm{B}^{\prime}$.)

However, as we have seen, both Theorems $\mathrm{B}^{\prime}$ and $\mathrm{C}^{\prime}$ are actually false if one merely assumes that the functions involved have only multiple zeros (i.e., zeros of multiplicity at least 2). Thus the path of reasoning sketched above is barred for Theorem 1. 


\section{A substitute for Theorem $\mathrm{C}^{\prime}$}

Although Theorem $\mathrm{C}^{\prime}$ fails for families of meromorphic functions all of whose zeros are multiple (but not necessarily of multiplicity greater than 2), we do have a substitute result. Recall that a family $\mathcal{F}$ of functions meromorphic on $D$ is said to be quasinormal on $D$ if from each sequence $\left\{f_{n}\right\} \subset \mathcal{F}$ one can extract a subsequence $\left\{f_{n_{k}}\right\}$ which converges (with respect to the spherical metric) locally uniformly on $D \backslash E$, where the set $E$ (which may depend on $\left\{f_{n_{k}}\right\}$ ) has no accumulation point on $D$. If $E$ can always be chosen to have no more than $m$ points, $\mathcal{F}$ is said to be quasinormal of order $m$ on $D$; cf. [5].

Theorem 2. Let $\mathcal{F}$ be a family of meromorphic functions on a plane domain $D$, all of whose zeros are multiple. If for each $f \in \mathcal{F}, f^{\prime}(z) \neq 1$ for all $z \in D$, then $\mathcal{F}$ is quasinormal of order 1 on $D$.

Theorem 2 extends the main result of [5], where it is assumed that the family $\mathcal{F}$ is quasinormal on $D$.

\section{Some AuXiLIARY RESUlts}

We require some notation. As before, $\Delta$ denotes the unit disc. More generally, $\Delta(a, r)$ is the open disc of radius $r$ and center $a$, and $\Delta^{\prime}(a, r)$ is the same disc minus its center.

Lemma 1. Let $\left\{f_{n}\right\}$ be a sequence of functions meromorphic on a plane domain $D$, all of whose zeros are multiple and such that $f_{n}^{\prime}(z) \neq 1$ for all $n$ and all $z \in \Delta$. Suppose that no subsequence of $\left\{f_{n}\right\}$ is normal at the point $a \in D$. Then there exists $\delta_{0}>0$ such that for any $0<\delta \leq \delta_{0}, f_{n}$ has only a single (multiple) zero on $\Delta(a, \delta)$ for all sufficiently large $n$.

The proof of Lemma 1 uses Theorem 2 above and Lemmas 3 and 4 of [5].

Lemma 2. Let $\left\{f_{n}\right\}$ be a sequence of functions meromorphic on $\Delta$, all of whose zeros are multiple, such that $f_{n}^{\prime}(z) \neq 1$ for all $n$ and all $z \in \Delta$. Suppose that

(a) $\left\{f_{n}\right\}$ is normal on $\Delta^{\prime}(0,1)$, but no subsequence of $\left\{f_{n}\right\}$ is normal at 0 ; and

(b) there exists $\delta>0$ such that $f_{n}$ has a single (multiple) zero on $\Delta(0, \delta)$ for all large $n$.

Then there exists a subsequence $\left\{f_{n_{k}}\right\}$ of $\left\{f_{n}\right\}$ such that for any $s \in \mathbb{C}, f_{n_{k}}-s$ has at most two zeros (counting multiplicity) on $\Delta(0,1 / 2)$.

This is Lemma 7 of [5].

Recall that the order of a meromorphic function $f$ on $\mathbb{C}$ is defined by

$$
\rho=\limsup _{r \rightarrow \infty} \frac{\log T_{0}(r)}{\log r}
$$

here $T_{0}(r)$ is the Ahlfors-Shimizu characteristic of $f$ defined by

$$
T_{0}(r)=\int_{0}^{r} \frac{S(t)}{t} d t,
$$

where

$$
S(t)=\frac{1}{\pi} \iint_{|z| \leq t}\left[f^{\#}(z)\right]^{2} d x d y .
$$


For $0<a<b$, set $S(a, b)=S(b)-S(a)$. If we wish to emphasize the function under consideration, we write $S(a, f)$ and $S(a, b ; f)$. Similarly,

$$
S(D, f)=\frac{1}{\pi} \iint_{D}\left[f^{\#}(z)\right]^{2} d x d y
$$

for any plane domain $D$.

We have the following simple result.

Lemma 3. If the order of the meromorphic function $f$ is nonzero, then there exist $r_{n} \rightarrow \infty$ such that $S\left(r_{n} / 2, r_{n} ; f\right) \rightarrow \infty$.

Proof. Otherwise, we have $S(r, f) \leq M \log r$ for some $M>0$ and all $r \geq 2$. Indeed, suppose that there exists $C>0$ such that $S(\rho / 2, \rho ; f) \leq C$ for all $\rho>0$. Let $r \geq 2$. Take $n=\left\lfloor\log _{2} r\right\rfloor$, so that $2^{n} \leq r<2^{n+1}$. Then since

$$
S\left(2^{j}, f\right) \leq S\left(2^{j-1}, f\right)+C, \quad j=1,2, \ldots,
$$

we have

$$
S(r, f) \leq S\left(2^{n+1}, f\right) \leq S\left(2^{n}, f\right)+C \leq \cdots \leq S(2, f)+n C \leq M \log r,
$$

where $M=(S(2, f)+C) / \log 2$. But then

$$
\begin{aligned}
T_{0}(r)=\int_{0}^{r} \frac{S(t)}{t} d t & \leq C_{f}+\int_{2}^{r} \frac{M \log t}{t} d t \\
& <C_{f}+\frac{1}{2} M(\log t)^{2},
\end{aligned}
$$

where $C_{f}=\int_{0}^{2} \frac{S(t)}{t} d t$ depends only on $f$; and so, by (1) and (2),

$$
\rho=\limsup _{r \rightarrow \infty} \frac{\log T_{0}(r)}{\log r}=0,
$$

which contradicts the assumption $\rho>0$.

\section{Proof of Theorem 1}

We now show how Theorem 1 can be derived from Theorem 2. Full proofs and generalizations will appear elsewhere.

Suppose, then, that $f$ is a transcendental meromorphic function of order $\rho$ on $\mathbb{C}$, all of whose zeros are multiple, and that $f^{\prime}(z)=1$ at most finitely often. Theorem $\mathrm{A}^{\prime \prime}$ shows that one cannot have $\rho<\infty$. We show that $\rho=\infty$ cannot hold either; in fact, we derive a contradiction from the assumption $\rho>0$.

Let $F(z)=f(z) / z$. Then $F$ also has order $\rho$, so by Lemma 3 , there exists $r_{n} \rightarrow \infty$ such that

$$
S\left(r_{n} / 2, r_{n} ; F\right) \rightarrow \infty
$$

Set

$$
f_{n}(z)=f\left(r_{n} z\right) / r_{n}
$$

and

$$
F_{n}(z)=f_{n}(z) / z=F\left(r_{n} z\right)
$$


Then for $1 / 2 \leq|z| \leq 1$,

$$
\begin{aligned}
F_{n}^{\#}(z) & =\frac{\left|z f_{n}^{\prime}(z)-f_{n}(z)\right|}{|z|^{2}+\left|f_{n}(z)\right|^{2}} \\
& \leq \frac{\left|z f_{n}^{\prime}(z)\right|}{|z|^{2}+\left|f_{n}(z)\right|^{2}}+\frac{\left|f_{n}(z)\right|}{|z|^{2}+\left|f_{n}(z)\right|^{2}} \\
& \leq 4 f_{n}^{\#}(z)+1 .
\end{aligned}
$$

We claim that $\left\{f_{n}\right\}$ is not normal on $D=\{z: 1 / 3<|z|<3\}$. For otherwise, by Marty's theorem, there exists $M^{\prime}>0$ such that $f_{n}^{\#}(z) \leq M^{\prime}$ for $z \in K=$ $\{z: 1 / 2 \leq|z| \leq 1\}$ and all $n$. By (6),

$$
F_{n}^{\#}(z) \leq 4 M^{\prime}+1=M
$$

for all $z \in K$. Since by (5),

$$
F_{n}^{\#}(z)=r_{n} F^{\#}\left(r_{n} z\right)
$$

the change of variable formula for multiple integrals together with (7) gives

$$
\begin{aligned}
S\left(r_{n} / 2, r_{n}, F\right) & =S\left(1 / 2,1, F_{n}\right) \\
& =\frac{1}{\pi} \iint_{K}\left[F_{n}^{\#}(z)\right]^{2} d x d y<M^{2},
\end{aligned}
$$

which contradicts (3).

So let $a \in D$ be a point at which $\left\{f_{n}\right\}$ fails to be normal; clearly, we may choose $a \in K$. Taking a subsequence and renumbering, we may assume further that no subsequence of $\left\{f_{n}\right\}$ is normal at $a$. Now $f_{n}^{\prime}(z)=f^{\prime}\left(r_{n} z\right)$ by (4), and $r_{n} z \rightarrow \infty$ uniformly on $D$. Thus $f_{n}^{\prime}(z) \neq 1$ for $z \in D$ and $n$ sufficiently large; so, dropping a finite number of terms and renumbering again, we may assume that $f_{n}^{\prime}(z) \neq 1$ on $D$ for all $n$. Since all zeros of $f_{n}$ are clearly multiple, it follows from Theorem 2 that $\left\{f_{n}\right\}$ is quasinormal of order 1 on $D$ and hence normal on $D \backslash\{a\}$.

By Lemma 1 , there exists $0<\delta<1 / 12$ such that $f_{n}$ has only a single (multiple) zero on $\Delta(a, \delta)$ for sufficiently large $n$. Applying Lemma 2 (with $\Delta(a, 1 / 6) \subset D$ in place of $\Delta)$, we obtain a subsequence, which we again denote $\left\{f_{n}\right\}$, such that for any $s \in \mathbb{C}, f_{n}(z)=s$ has at most two solutions (counting multiplicity) in $\Delta(a, \delta)$. Since $S(D, g)$ is the normalized spherical area of the image of $D$ under $g$ (counting multiplicities),

$$
S\left(\Delta(a, \delta), f_{n}\right) \leq 2
$$

for all $n$. On the other hand, since $\left\{f_{n}\right\}$ is normal on $D \backslash\{a\}$, the functions $f_{n}^{\#}$ are uniformly bounded on $K \backslash \Delta(a, \delta)$, so that

$$
S\left(K \backslash \Delta(a, \delta), f_{n}\right) \leq C
$$

for some $C>0$ and all $n$. It follows from (8) and (9) that for all $n$,

$$
\begin{aligned}
\frac{1}{\pi} \iint_{K}\left[f_{n}^{\#}(z)\right]^{2} d x d y & \leq S\left(K \backslash \Delta(a, \delta), f_{n}\right)+S\left(\Delta(a, \delta), f_{n}\right) \\
& \leq C+2 .
\end{aligned}
$$


As before, we have by (5) and (6),

$$
\begin{aligned}
S\left(r_{n} / 2, r_{n} ; F\right) & =S\left(1 / 2,1, F_{n}\right)=\frac{1}{\pi} \iint_{K}\left[F_{n}^{\#}(z)\right]^{2} d x d y \\
& \leq \frac{1}{\pi} \iint_{K}\left[4 f_{n}^{\#}(z)+1\right]^{2} d x d y
\end{aligned}
$$

for all $n$. Expanding the integrand on the right-hand side of (11), applying the Cauchy-Schwarz inequality, and invoking (10), we obtain a contradiction to (3). This completes the proof of Theorem 1.

\section{REFERENCES}

1. W. Bergweiler and A. Eremenko, On the singularities of the inverse to a meromorphic function of finite order, Rev. Mat. Iberoamericana 11 (1995), 355-373. MR.1344897 (96h:30055)

2. H. H. Chen and M. L. Fang, On the value distribution of $f^{n} f^{\prime}$, Sci. China Ser. A 38 (1995), 789-798. MR1360682 (97a:30035)

3. W. K. Hayman, Picard values of meromorphic functions and their derivatives, Ann. of Math. (2) 70 (1959), 9-42. MR0110807 (22:1675)

4. Y. X. Ku, Un critère de normalité des familles de fonctions méromorphes, Sci. Sinica Special Issue 1 (1979), 267-274. (Chinese) MR0662205 (83i:30047)

5. X. C. Pang, Sh. Nevo, and L. Zalcman, Quasinormal families of meromorphic functions, Rev. Mat. Iberoamericana 21 (2005), 249-262. MR2155021 (2006d:30046)

6. Y. F. Wang and M. L. Fang, Picard values and normal families of meromorphic functions with multiple zeros, Acta Math. Sinica (N.S.) 14 (1998), 17-26. MR.1694044 (2000g:30026)

7. L. Zalcman, On some questions of Hayman, unpublished manuscript, 1994.

8. L. Zalcman, Normal families: new perspectives, Bull. Amer. Math. Soc. (N.S.) 35 (1998), 215-230. MR1624862 (99g:30048)

Department of Mathematics, Bar-Ilan University, 52900 Ramat-Gan, Israel

E-mail address: nevosh@macs.biu.ac.il

Department of Mathematics, East China Normal University, Shanghai 20062, P. R. CHINA

E-mail address: xcpang@euler.math.ecnu.edu.cn

Department of Mathematics, Bar-Ilan University, 52900 Ramat-Gan, Israel

E-mail address: zalcman@macs.biu.ac.il 